\title{
Approaches Half Band Filter Realization for Means FPGA
}

\author{
Oleksandr Vorgul \\ Department of Microprocessor Technologies and Systems \\ Kharkiv National University of Radio Electronics \\ Kharkiv, Ukraine \\ oleksandr.vorgul@nure.ua
}

\begin{abstract}
Approaches to half band filter realization are considered. Ways of implementation are discussed. Possibilities for obtaining FIR or IIR realizations are mentioned.
\end{abstract}

Keywords-FPGA, half band filter, digital signal processing, analytical signal

\section{INTRODUCTION}

When solving problems of synthesis and analysis of systems of different purposes, imitative modeling plays an increasingly important role. The current level of development of hardware and software allows you to perform modeling work at different levels of equipment from the idea of the system, expressed in mathematical form to its implementation. In itself, the simulation in this case is not the ultimate goal of this work, but is an instrument in the course of designing and allows us to conveniently evaluate the quality of the system from different points of view [1].

The object of the study is a half-band filter, which is being developed in digital form. Such a filter is extremely useful for the developer, since it is the basic element for performing many operations on a signal - frequency transfer, modulation, demodulation, to name a few. It is also convenient because it allows you to perform quality control work both in frequency and time characteristics, as well as in key parameters that are relevant to the researcher's current task, such as the number of valves, the value of the delay, etc.

Since the filter is digital, using the Matlab and Simulink software, you can perform simulations, from the task of filter parameters to its implementation, which is very convenient, cheap and really consumes a minimum of time.

Matlab allows you to get the filter structure of the given kind, its coefficients taking into account or without rounding off, as well as in a convenient form to present a complete set of filter characteristics in the shortest possible time and qualitatively [2]. Unfortunately, for an analog filter the task is more difficult to solve.

But to implement the filter in the FPGA, you need a convenient transition to the chosen environment of the description of digital equipment - VHDL, Verilog, System Verilog. Thus, the design is already in the specialized program (Quartus II or Vivado) and is close to the apparatus synthesis $[3,4]$. This transition allows Matlab to be executed in semi-automatic mode. This adds flexibility to the design process and allows you to reduce the cost of design.

A bit of technical characteristics. Bit length - no less than 16 bits. What element base was available for research?

1. Spartan 6. $45 \mathrm{~nm}$. The limit on the digit is rather vague. On the crystal, high-speed hardware multipliers $18 \times 18$ bits and a 48-bit battery are implemented, which significantly but not completely limits the bit. In principle, you can increase the digit. The charge on the crystal, of course, increases, but can be used for educational purposes.

2. Artix 7. Compared to it, Spartan looks simple. In addition to offering Xilinx on the Spartan 6 example for the initial level of familiarity with the capabilities of modern FPGAs, it is possible to master the interfaces that are widely used at present. Actually, for work with interfaces Artix 6 and optimized. These include interfaces such as PCI Express Base Spec 2.1 Gen1 (2.5 Gb / s) and Gen2 (5 Gb / s). Maximum speed up to $6.25 \mathrm{~Gb} / \mathrm{s}$. It is possible to convert the serial code into parallel words in the length of 16, 20, 32, or 40 bits in the receiver and perform a reverse operation by the transmitter.

\section{CAlculation OF THE CoEFFICIENTS}

Half-band filters are lowpass FIR filters with cut-off frequency of one-quarter of sampling frequency $\mathrm{f}_{\mathrm{s}}$ and odd symmetry about $f_{s} / 4$ [5]. And it so happens that almost half of the coefficients are zero. The passband and stopband bandwiths are equal, making these filters useful for decimation-by-2 and interpolation-by-2. Since the zero coefficients make them computationally efficient, these filters are ubiquitous in DSP systems.

Here we will compute half-band coefficients using the window method. While the window method typically does not yield the fewest taps for a given performance, it is useful for learning about half-band filters. Efficient equiripple halfband filters can be designed using the Matlab function firhalfband [5].

\section{COEFFICIENTS BY THE WINDOW METHOD}

The impulse response of an ideal lowpass filter with cutoff frequency $\omega_{c}=2 \pi f_{c} / f_{s}$ is [5]:

$$
\mathrm{h}(\mathrm{n})=\sin \left(\omega_{\mathrm{c}} \mathrm{n}\right) / \pi \mathrm{n},-\infty<\mathrm{n}<\infty .
$$


This is the familiar $\sin x / x$ or sinc function (scaled by $\left.\omega_{\mathrm{c}} / \pi\right)$. To create a filter using the window method, we truncate $\mathrm{h}(\mathrm{n})$ to $\mathrm{N}+1$ samples and then apply a window. For a halfband filter, $\omega_{\mathrm{c}}=2 \pi^{*} 1 / 4=\pi / 2$. So the truncated version of $h(n)$ is:

$$
\mathrm{h}(\mathrm{n})=\sin (\mathrm{n} \pi / 2) / \mathrm{n} \pi,-\mathrm{N} / 2<\mathrm{n}<\mathrm{N} / 2 .
$$

Now apply a window function $w(n)$ of length $N+1$ to obtain the filter coefficients $b$ :

$$
\begin{aligned}
& \mathrm{b}(\mathrm{n})=\mathrm{h}(\mathrm{n}) \mathrm{w}(\mathrm{n}),-\mathrm{N} / 2<\mathrm{n}<\mathrm{N} / 2, \\
& \mathrm{~b}(\mathrm{n})=\sin (\mathrm{n} \pi / 2) /(\mathrm{n} \pi) * \mathrm{w}(\mathrm{n}),-\mathrm{N} / 2<\mathrm{n}<\mathrm{N} / 2 .
\end{aligned}
$$

Choice of a particular window function $\mathrm{w}(\mathrm{n})$ is based on a compromise between transition bandwidth and stopband attenuation. As a simple example, the Hamming window function of length $\mathrm{N}+1$ is:

$$
\mathrm{w}(\mathrm{n})=0.54+0.46 \cos (2 \pi \mathrm{nN}),-\mathrm{N} / 2<\mathrm{n}<\mathrm{N} / 2
$$

And this way you can obtain the coefficients. Note that $\mathrm{N}$ is even and the filter length is $\mathrm{N}+1$ (odd length).

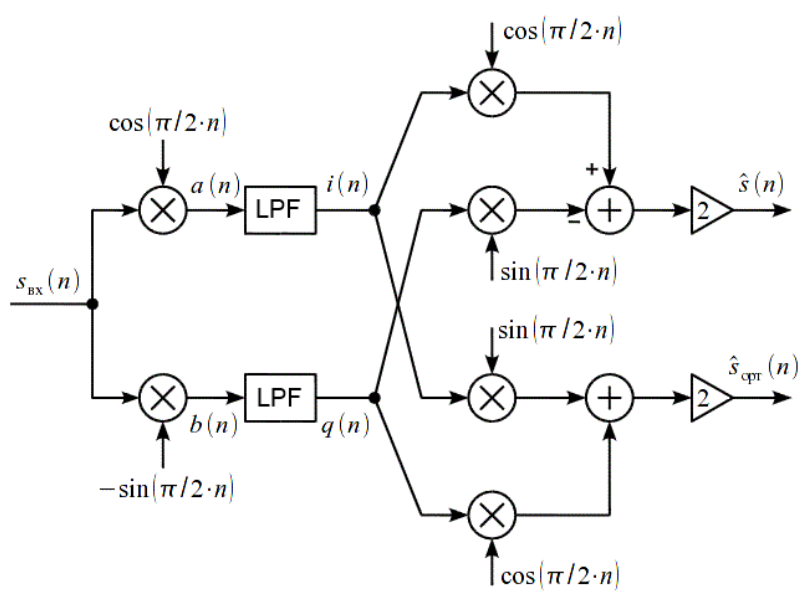

Fig. 1. Analytical signal shaper based on quadrature converter.

\section{BRIEF RESULTS OF WORK}

The half band filter is supposed to implement for analytical signal forming. The main task in the formation of the analytical signal is the suppression of the spectral components in the negative frequency range falls on the halfband low-pass filter. One can use either FIR or IIR filters. When using IIR filters, one can save computational resources, and calculate the filter based on the required frequency response corridor. However, the use of a physically realized low-pass filter will lead to signal distortion in the low and high frequencies
Real low-pass filter has non-uniformity in the passband (in the positive frequency range), possesses finite suppression in the negative-frequency range, and some transition band, with the result that the signal in the low and high frequencies is distorted. But for practical use of such a shaper for SSB modulation during voice transmission, distortions in the low and high frequency areas do not play a significant role, while the use of, for example, elliptical IIR filters allows you to adjust the level of sideband suppression and uneven frequency response.

Thus, all multiplications can be replaced by re-indexing the samples and changing their sign. This structure requires only multiplication for filtering, which makes it attractive both for software implementation and for hardware based on FPGA.

The concepts of a bandpass signal and a complex radio signal envelope are used, and the concept of a vector representation of a complex envelope makes possible to realize modulation and demodulation under general schemes. The complex envelope can be represented by the in-phase and quadrature components and the modulation can be performed by a quadrature modulator. In order to specify the modulation method, it is necessary to choose the method of forming the complex signal envelope by changing the amplitude and phase.

The project in Matlab is a half band filter of the 32nd and 128th order. When switching to Vivado, we have the opportunity to quickly obtain a filter structure and the ability to explore the characteristics of the digital system. Considering such a simple example, we demonstrate the possibilities of interacting with different products. The power of crystals is enough to implement even an optimized algorithm: about 1100 instances and 320 nodes (DFlops) require an algorithm where the weakest version of Spartan has 3840 elements (Logic Cells), as well as Artix 7. More detailed results are given in the work.

\section{REFERENCES}

[1] Moiseev N.N. Mathematical problems of system analysis: [Proc. manual for universities on spec. "Apply Mathematics"] / N. N. Moiseev. - M .: Science, 1981. - 487 p.

[2] Digital signal processing and MATLAB: proc. manual / A.I. Solonina, D.M. Klionsky, T.V. Merkucheva, S.N. Perov. - SPb .: BHV-Petersburg, 2013. - 512 pp., Ill .- (Educational literature for universities)

[3] Semenets V. V. Designing digital systems using the VHDL language: studies. manual / V.V. Semenets, I.V. Khakhanova, V.I. Khakhanov; MES of Ukraine, KNURE. - Kharkov: KNURE, 2003. - 492 p.

[4] Semenets V. Century Technology of interconnection of electronic equipment: studies. for universities / V. V. Semenets, John Kratz, I. Sh. Nevludov, V. A. Palagin. - H.: ed. SMITH, 2005. - 432 p. [Semenets V. V., Kratts J., Nevlyudov I. Sh., Palagin V. A].

[5] Neil Robertson. Simplest Calculation of Half-band Filter Coefficients. https://www.dsprelated.com/blogs.php 